\title{
Xilotecnia de la madera de Schinus molleL. de una plantación forestal comercial en Hidalgo, México
}

\author{
Xilotecnia of the wood of Schinus molle L. of a commercial forest \\ plantation in Hidalgo, Mexico
}

\author{
Guadalupe Olvera-Liconal, Roberto Machucal, Amparo Borja '*, Alejandro Corona', Idalia Zaragozal, \\ Jesús Guadalupe Arreola ${ }^{2}$ y Javier Jiménez'
}

1 Universidad Autónoma Chapingo. División de Ciencias Forestales. Texcoco, Estado de México, México.

\author{
2 Universidad Autónoma Chapingo. Unidad Regional \\ Universitaria de Zonas Áridas. Bermejillo, Durango \\ México. \\ * Autor de correspondencia. aborja@correo.chapingo.mx
}

\section{Resumen}

El presente trabajo tuvo como objetivo determinar las características anatómicas, las propiedades físicas, así como estimar las propiedades mecánicas, de la madera de cuatro árboles de Schinus molle L. (pirú) provenientes de una plantación forestal comercial en Tolcayuca, Hidalgo, México. El estudio se realizó en el Laboratorio de Anatomía y Tecnología de la Madera de la División de Ciencias Forestales de la Universidad Autónoma Chapingo. Como resultado se obtuvo que el color de la madera en la albura es rosado, en el duramen es de color café. La madera no tiene olor y sabor característicos, es de brillo medio, veteado pronunciado, hilo inclinado y textura media. La porosidad es semidifusa y el parénquima axial difuso y escaso. Los radios son uniseriados, biseriados y triseriados. Los vasos son cortos, muy abundantes, las fibras son medianas y finas. Se obtuvo una densidad básica de 0.54 y poder calorífico de $17.246 \mathrm{MJ} \mathrm{kg}^{-1} \mathrm{La}_{\text {dureza }}$ lateral y en extremos (longitudinal) en condición verde se clasificó como media y baja respectivamente, a 12\% de contenido de humedad, se clasificó como media y alta respectivamente; la madera de pirú se clasificó como muy buena para pulpa y papel. La contracción volumétrica total se clasificó como media, la contracción tangencial total: muy alta y la contracción radial total: alta. La relación de anisotropía se clasificó como alta. El punto de saturación de la fibra se describió normal. Por sus características y propiedades de la madera se puede utilizar en la fabricación de muebles, mangos de herramientas y pisos.

PALABRAS CLAVE: anatomía, contracción, densidad, pirú, pirul, poder calorífico.

\section{Abstract}

The objective of this work was to determine the anatomical characteristics, physical properties, and estimate the mechanical properties of wood of four trees of Schinus molle L. (pirú) from a commercial forest plantation in Tolcayuca, state of Hidalgo, Mexico. The study was carried out in the Wood Anatomy and Technology Laboratory at the Division of Forestry Sciences at the Chapingo Autonomous University. As a result, it was found that the color of the wood in the sapwood is pink, the heartwood is brown. The wood has no characteristic smell and flavor; ita has an average shine, pronounced grain figure, inclined fiber direction, and medium texture. The porosity is semi-diffuse and the axial parenchyma diffuse and scarce. The rays are uniseriate, biseriate and triserial. The vessels are short and abundant, the fibers are medium size and thin. The basic density is 0.54 and the calorific value of $17.246 \mathrm{MJ} \mathrm{kg}^{-1}$. The lateral and extreme hardness in green condition was classified as medium and low respectively. By Runkel relationship pirul wood is classified as very good for pulp and paper. The total volumetric contraction is classified as medium. The total tangential contraction: very high and the total radial contraction: high. The anisotropy relation is classified as high. The fiber saturation point of the is classified as normal.

KEYWORDS: anatomy, shrinkage, density, pirú, pirul, calorific value. 


\section{INTRODUCCIÓN}

El Schinus molle es una especie exótica muy común en México, nativa de Sudamérica; fue introducida en el Valle de México por Antonio de Mendoza y Pacheco, Virrey de la Nueva España entre los años 1535 y 1550. (Corkidi, Cacho y Búrquez, 1991; Ramírez-Albores y Badano, 2013). Es una de las 15 especies que ocurren desde México hasta Chile, una de las más abundantes en el Nuevo Mundo (Goldstein y Coleman, 2004). Esta especie se distribuye en la Altiplanicie y Mesa Central, crece de modo silvestre en clima subhúmedo o árido, donde subsiste a pesar de las heladas, sequias y suelos erosionados o salinos. (Camacho y Ramírez, 1987). En sus áreas de origen y de mayor distribución (Perú y Bolivia) es considerado como un árbol de uso múltiple (Carrere, 2009).

En la agricultura tiene un valor integral (económico, social y cultural), es proveedor de leña y es útil para la conservación de suelos, lo que lo hace importante como componente agroforestal. (Whaley et al., 2010; Brandt, Zimmermann, Hensen, Castro y Rist, 2012)

También existen varios estudios en relación con la extracción de aceites esenciales de hojas y frutos en los cuales se determina la actividad antifúngica, antimicrobiana antioxidante anticancerígena, antidepresiva e insecticida (Molina-Salinas et al., 2007; Mohareb, Badawy y Abdelgaleil, 2013; López et al., 2014; Martins, Arantes, Candeias, Tinoco y Cruz-Morais, 2014 y dos Santos et al., 2015).

Igualmente, existen registros sobre Schinus johnstonii y S. matchandii, las cuales son aprovechadas en el noreste de la Patagonia Argentina como combustibles, consideradas entre las cuatro especies preferidas (Cardoso, Ladio y Lozada, 2012; Cardoso, Ladio y Lozada, 2013; Cardoso, Ladio, Dutrus y Lozada, 2015). Schinus spp.: Schinus longifolia (Lindl.) Speg.var. longifolia/Schinus fasciculatus (Griseb.) I.M. Johnst. var. fasciculatus se encuentra entre las 15 especies más prominentes para utilizarse como leña en el cocimiento de alimentos (Martínez, 2015).

En comunidades rurales de México, las hojas del $S$. molle (pirú) se usan en baños de vapores purificadores conocidos como Temazcal; además, el aceite esencial tiene propiedades antisépticas, antiespasmódicas y sedantes, estimula la secreción gástrica, proporciona estimulación uterina, funciona como antiinflamatorio en casos de cervicitis y vaginitis, además tiene acción inhibitoria frente a bacterias, hongos y levaduras (Ramírez-Albores y Badano, 2013; Alba, Bonilla y Arroyo, 2009).

El pirú también es apreciado como especie ornamental, por su rápido crecimiento bajo condiciones de intensa radiación solar, para establecer cortinas rompevientos, como sombra para el ganado, en la reforestación de áreas muy degradadas por soportar sequias, heladas, suelos ligeramente salinos. Adicionalmente, representa una opción potencial para la apicultura pues produce cuantiosas cantidades de néctar, por su largo periodo de floración (Camacho, 1985; Ramírez-Albores y Badano, 2013).

En relación con la madera del pirú, existen estudios relacionados con anatomía macroscópica y microscópica (Islas, 1986; Schulte, 1992), densidad verde, densidad seca al aire libre, densidad básica, contracciones totales lineales y contracción volumétrica, relación de anisotropía, dureza, compresión perpendicular y cizallamiento (Schulte, 1992; Luna, 2012). También se cuenta con estudios de pH, extractivos, holocelulosa y lignina (Schulte, 1992; MejíaDíaz y Rutiaga-Quiñones, 2008). La madera de S. molle L. se utiliza en postes, pisos interiores, implementos de granja, tales como arados, yugos y mangos de herramientas; muebles rústicos, construcción, combustibles (Camacho y Ramírez, 1987; Aderson, s.f.).

\section{ObJetivos}

El objetivo de este estudio fue conocer las características anatómicas y las propiedades físicas, así como calcular los índices de las propiedades mecánicas de la madera de Schinus molle L. proveniente de una plantación del estado de Hidalgo, México para prevenir un mal uso del recurso o simplemente su inutilización. 


\section{MATERIALES Y MÉTODOS}

\section{Descripción de la especie}

Schinus molle L. pertenece a la familia Anacardiaceae, tiene varias sinonimias: Schinus buingán Molina, Schinus molle var. buingan, Schinus molle var. areira (L.) DC., Scbinus angustifolius Sessé \& Moc., Schinus bituminosus L., Schinus occidentalis Sessé \& Moc., Schinus areira L. (Luna, 2012). Igualmente, tiene varios nombres comunes: pirul, pirú, árbol del Perú (México); molle, cuyash, huaribay (Perú); aymara (Bolivia); muelle, falso pimiento, pimiento (Colombia); mulli (Ecuador); pimenteiro, aroeira, amescla, aroeira-periquita, bálsamo, fruto de sabiá (Brasil); aguaribay, terebinto, árbol de la pimienta, gualeguay (Argentina); pimiento (Chile). Otros nombres comunes en México: Pirwi, Tsactumi, Tzactumi, Tzantuni (Rep. Mex.); pirú, pirul (Valle de México); Xasa, Xaza (I. Otomî); Peloncuáhuitl (I. Náhuatl) Yaga-cica, Yaga-lache (I. zapoteca, Oax.) (Luna, 2012).

Schinus molle es originario de la región andina de Sudamérica, principalmente Perú. Crece en altitudes de hasta $3650 \mathrm{~m}$ y es ampliamente distribuido en Centroamérica, en Estados Unidos (sur de California y oeste de Texas), en Argentina (Corrientes, Entre Ríos, Misiones, Salta y Santa Fe) (Luna, 2012). En México, se distribuye en Aguascalientes, Chiapas, Coahuila, Distrito Federal, Durango, Guanajuato, Guerrero, Hidalgo, Estado de México, Jalisco, Michoacán, Morelos, Nuevo León, Oaxaca, Puebla, Querétaro, San Luís Potosí, Sinaloa, Tlaxcala, Veracruz y Zacatecas (Rzedowski y Calderón, 1999; Villaseñor y Espinosa, 1998). Schinus molle es un árbol polígamo dioico, alcanza alturas entre $6 \mathrm{~m}$ y $15 \mathrm{~m}$; en ocasiones alcanza $25 \mathrm{~m}$., diámetro entre $30 \mathrm{~cm}$ y $80 \mathrm{~cm}$, pudiendo alcanzar hasta $1.5 \mathrm{~m}$. El tallo por lo general es torcido, resinoso, rugoso al tacto: la corteza es áspera más bien escamosa, hojas alternas, regularmente imparipinnadas la inflorescencia es una panícula, flores pequeñas, con simetría radial, de color amarillo-verdoso a blanquecinas, unisexuales pero frecuentemente con rudimentos del otro sexo en las flores femeninas, los estambres están reducidos y las anteras vacías y, en las flores masculinas, el ovario es rudimentario, el fruto tiene forma de drupa, es pequeño, carnoso color rojo, brillante, con una sola semilla. (Schulte, 1992; Calderón y Rzedowski, 2001; Rzedowski y Calderón, 1999).

\section{Descripción del Área de Estudio}

El área de estudio está ubicada en el municipio de Tolcayuca, estado de Hidalgo, se localiza en las coordenadas geográficas $19^{\circ} 57^{\prime} 09^{\prime}$ ' latitud Norte, $98^{\circ} 56^{\prime}$ 53.7" longitud Oeste, altitud de $2404 \mathrm{~m}$. Colinda al norte con San Agustín Tlaxiaca y Zapotlán, al sur con Tizayuca y Estado de México, al este con Tezontepec y Zapotlán, y al oeste con el Estado de México (Fig. 1).

Presenta un clima templado, con temperatura media anual de $16.2^{\circ} \mathrm{C}$, precipitación pluvial de $557 \mathrm{~mm}$. El suelo es de tipo semidesértico. El municipio es atravesado por el rio Panuco y el río Moctezuma. La vegetación está formada de matorrales inermes y espinosos como el cardón, el maguey, el nopal, el pirú y las yucas. La fauna está integrada por conejos, coyotes, lobos, zorrillos y ardillas, reptiles, insectos, arácnidos, canarios, chupamirtos, gorriones, jilgueros y pájaros (Instituto Nacional para el Federalismo y el Desarrollo Municipal [INFDM], 2002).

\section{Colecta del material}

La colecta del material se realizó el día 15 de mayo de 2015. El terreno tiene una pendiente de $15 \%$ y se ubica en las coordenadas: $19^{\circ} 57^{\prime} 09^{\prime \prime}$ latitud norte, $98^{\circ}$ 56' 53.7" longitud oeste, altitud de $2404 \mathrm{~m}$. Se seleccionaron cuatro árboles de fuste recto, sanos y sin protuberancias, a cada árbol seleccionado se le midió la altura total y diámetro normal (Tabla 1). Así mismo se colectaron muestras botánicas con la finalidad de corroborar la especie. Posteriormente, los árboles se derribaron y trocearon, las trozas fueron trasladadas al laboratorio de plantas piloto de la División de Ciencias Forestales de la Universidad Autónoma Chapingo en el Estado de México. 


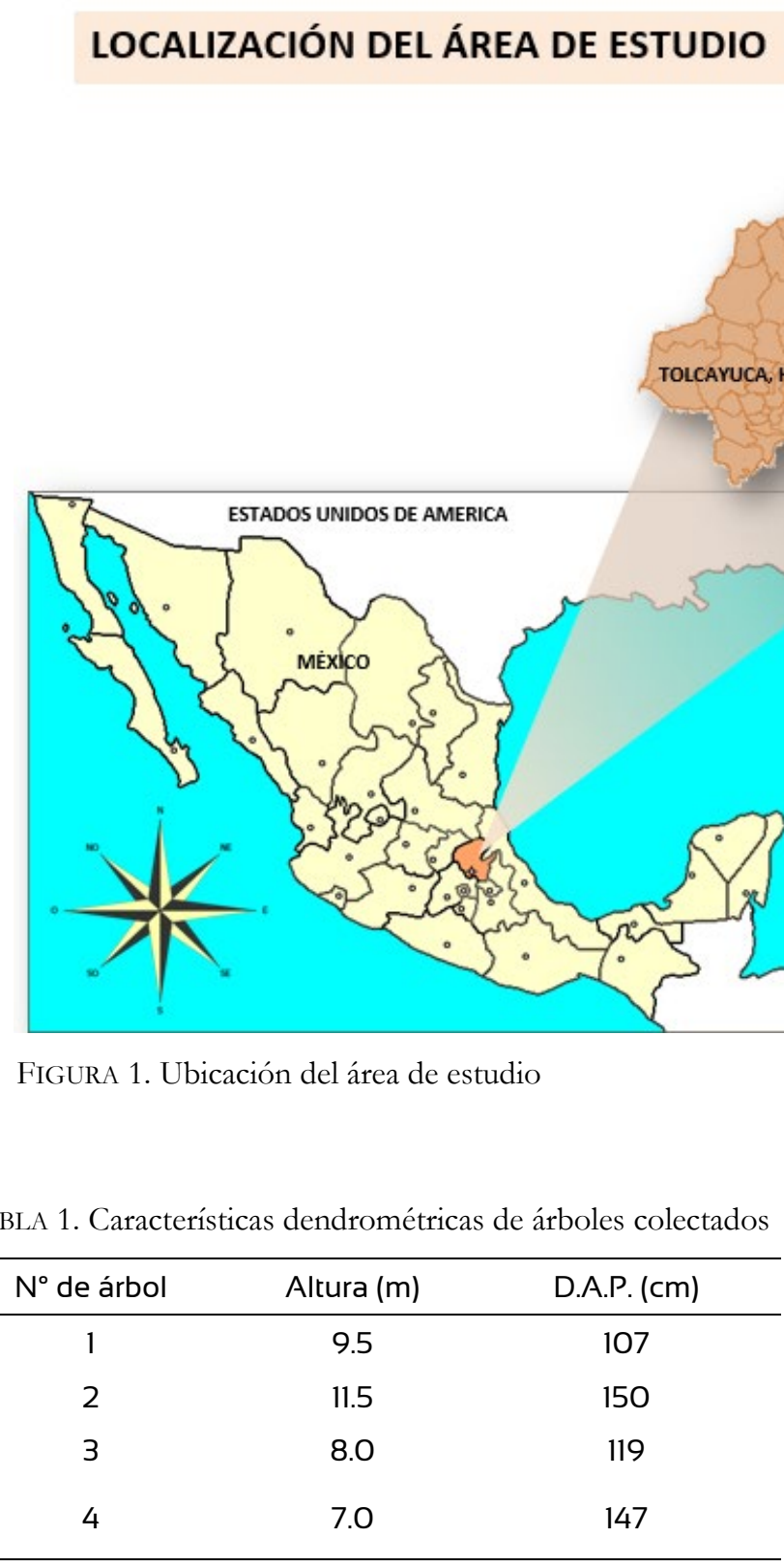

D.A.P. = Diámetro a $1.30 \mathrm{~m}$.

\section{Obtención de muestras de cada árbol}

De cada uno de los árboles, se obtuvieron rodajas para la elaboración de probetas; de la primera troza se obtuvo una rodaja de $5 \mathrm{~cm}$ de grosor para la descripción de características anatómicas microscópicas, otra de $5 \mathrm{~cm}$ para la determinación de las diferentes densidades y, finalmente, tres de $10 \mathrm{~cm}$ para calcular la contracción radial, tangencial y axial. Del resto de la troza se obtuvieron tablillas para la caracterización anatómica macroscópica (Fig. 2).

\section{Estudio anatómico}

Se elaboraron tablillas de $1 \mathrm{~cm} \times 7 \mathrm{~cm} \times 15 \mathrm{~cm}$ de los cortes típicos (tangencial, radial y transversal), en las cuales se realizaron observaciones a simple vista y con lupa de 10x de las características macroscópicas (color, sabor, olor, brillo, hilo, textura y veteado). Se clasificaron de acuerdo con Tortorelli (1956), el color se determinó según Munsell (2000).

En la rodaja de $5 \mathrm{~cm}$ se marcaron probetas de $2 \mathrm{~cm} \times$ $2 \mathrm{~cm} \times 2 \mathrm{~cm}$. Posteriormente, se obtuvieron cortes típicos transversales, longitudinales tangenciales y longitudinales radiales a un espesor de $20 \mu \mathrm{m}$ y materiales macerados (Navarro, Borja y Machuca, 2005). Los elementos constitutivos se midieron con el software LAS Core, usando los objetivos 5x, 10x y 40x., se determinó el tamaño de muestra con base en la longitud y ancho de las fibras y longitud de los vasos, mediante la ecuación 1. 
Troza de $1.5 \mathrm{~m}$.

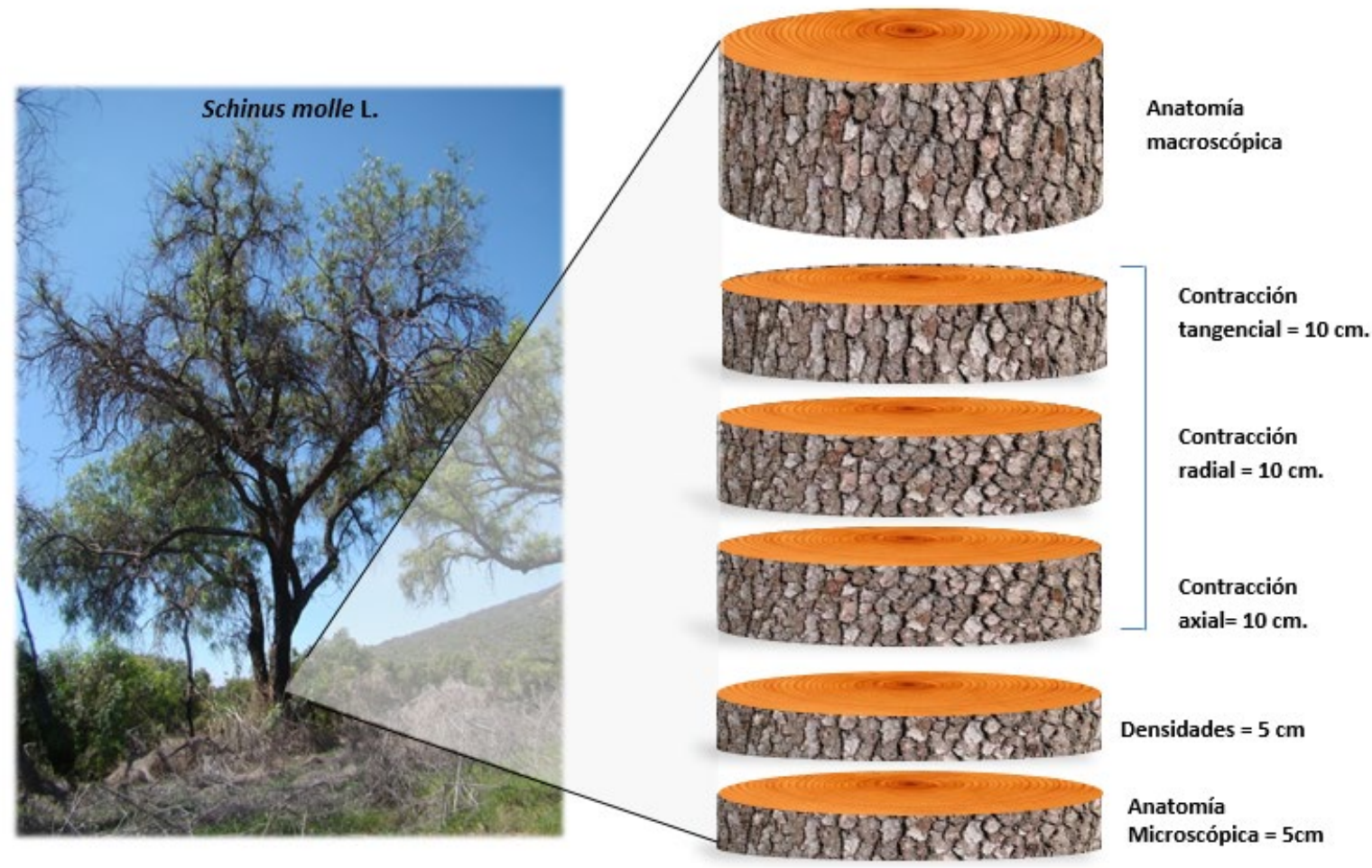

FIGURA 2. Obtención de rodajas para la elaboración de probetas.

$$
n=\frac{t^{2} c v^{2}}{E^{2}}
$$

Donde:

$n=$ tamaño de la muestra

$t=$ valor de la tabla de Student para una $\alpha=0.05$

$E=$ error relativo $=0.05$

$c v=$ coeficiente de variación

Se utilizó una premuestra de 36 fibras y 20 vasos de albura y de duramen. Los tamaños de muestra fueron 40 para fibras de albura y de duramen y 60 para vasos de albura y de duramen para cada árbol.

\section{Proporción de elementos constitutivos}

La determinación de la proporción de elementos constitutivos: fibras, vasos, radios y parénquima axial de la madera de pirú; se realizó en el corte transversal, en el cual se obtuvieron 450 fotografías con el objetivo 10x., con ayuda del Software LAS Core y con el Software ImageTool. Las mediciones de porcentaje de los elementos constitutivos se clasificaron de acuerdo con Kollmann (1959).

\section{Proporción de elementos de vaso}

Se obtuvo el área de los vasos existentes en el área determinada (considerando todas las formas y tamaños de los elementos de vasos). La proporción de elementos de vaso (PV), se calculó con la ecuación 2.

$$
P V(\%)=\frac{\text { Área de los elementos vaso }}{\text { Área determinada }} * 100
$$

\section{Proporción de radios}

En el área fijada se calculó el área de los radios existentes, la proporción de radios (PR) se determinó con la ecuación 3. 


$$
P R(\%)=\frac{\text { Área de radios }}{\text { Área determinada }} * 100
$$

\section{Proporción de parénquima axial}

Se obtuvo el área del parénquima axial (PPA) en el área determinada, se calculó la proporción de parénquima axial con la ecuación 4.

$$
\operatorname{PPA}(\%)=\frac{\text { Área de parénquima axial }}{\text { Área determinada }} * 100
$$

\section{Proporción de fibras}

La proporción de fibras (PF) para el área determinada se calculó por diferencia, con las proporciones antes calculadas (ecuación 5).

$$
P F(\%)=(100-P V-P R-P P A)
$$

$P V=$ proporción de elemento de vaso

$P R=$ proporción de radios

$P P A=$ proporción de parénquima axial

\section{Índices de calidad de pulpa para papel}

Con base en los datos obtenidos de longitud, diámetro de lumen y diámetro total de las fibras, se calcularon los índices de calidad de pulpa de acuerdo con Tamarit y Fuentes (2003).

\section{Propiedades físicas}

El contenido de humedad en condición verde ( $\mathrm{CHV})$, saturado y en equilibrio, se determinaron en 400 probetas de $2 \mathrm{~cm} \times 2 \mathrm{~cm} \times 2 \mathrm{~cm}$ (100 probetas de cada árbol) según Navarro et al. (2002). Las diferentes densidades: saturada, básica, anhidra, en equilibrio y normal, se calcularon de acuerdo con la Norma NOM EE-117-1981.

La densidad a $12 \%$ de contenido de humedad se determinó de la siguiente manera: en el laboratorio se registró el contenido de humedad $(\mathrm{CH})$, que fue de 14\%, este valor se tomó como referencia para calcular el incremento de $1 \%$ de peso, desde el peso anhidro hasta el peso a $14 \%$ de $\mathrm{CH}$ (ecuación 6).

$$
\Delta P 1 \%=\frac{P_{14}-P_{0}}{14}
$$

Donde:

$\Delta P 1 \%=$ Incremento de $1 \%$ de peso

$P_{14}=$ Peso al $14 \%$ de contenido de humedad

$P_{0}=$ Peso anbidro

Enseguida, se calculó el incremento del peso hasta 12\% de $\mathrm{CH}$ y, para el volumen a $12 \%$ de $\mathrm{CH}$ (ecuación 7 y 8 ), se siguió el mismo procedimiento indicado en Serna, Borja, Fuentes y Corona (2011).

$$
\Delta P_{12}=P_{0}+12(\Delta P 1 \%)
$$

Donde:

$\Delta P_{12}=$ Incremento de $12 \%$ de peso

$P_{0}=$ Peso anbidro

$\Delta P 1 \%=$ Incremento de $1 \%$ de peso

$$
\Delta V_{12}=V_{0}+12(\Delta V 1 \%)
$$

Donde:

$\Delta V_{12}=$ Incremento de $12 \%$ de volumen

$V_{0}=$ Volumen anhidro

$\Delta V 1 \%=$ Incremento de $1 \%$ de volumen

Las contracciones volumétricas, radiales, tangenciales y axiales, totales y parciales se calcularon de acuerdo con la norma mexicana NMX-EE-167-1983. Para calcular el punto de saturación de la fibra (PSF), se utilizaron los valores de densidad básica y contracción volumétrica total determinados previamente, se aplicó la ecuación 9, señalada por Tamarit y Fuentes (2003).

$$
P S F=\frac{\% \beta V t}{D b \times 0.9}
$$

Donde:

PSF $=$ Punto de saturación de la fibra

$\% \beta V t=$ Contracción volumétrica total

$\mathrm{D} b=$ Densidad básica 


\section{Coeficiente de contracción volumétrica}

Se refiere a la contracción que presenta la madera por cada $1 \%$ de contenido de humedad que esta disminuye, a partir del PSF, reflejando parte del grado de estabilidad dimensional de la madera. El coeficiente de contracción volumétrica se determinó con la ecuación 10.

$$
V \beta v=\frac{\% \beta V t}{P S F}
$$

Donde:

$V \beta v=$ coeficiente de contracción volumetrica

$\beta V t=$ contracción volumetrica total

$P S F=$ punto de saturación de la fibra

\section{Relación de anisotropía}

Es un valor que permite inferir el grado de estabilidad dimensional de la madera, representa la relación entre la magnitud de la contracción tangencial total y la magnitud de la contracción radial total. La relación de anisotropía se expresa en la ecuación 11.

$$
A=\frac{\% \beta T t}{\% \beta R t}
$$

Donde:

A = relación de anisotropía
$\% \beta T t=$ contracción tangencial total

$\% \beta \mathrm{R} t=$ contracción radial total

\section{Poder calorífico}

El poder calorífico de $S$. molle se determinó con un calorímetro PARR 6200, el cual cuantifica las calorías de acuerdo con la metodología del manual de operación del calorímetro del Laboratorio de Anatomía y Tecnología de la Madera de la División de Ciencias Forestales.

\section{Propiedades mecánicas}

Las propiedades mecánicas se calcularon de acuerdo con las fórmulas publicadas por Brown y Panshin (1980).

\section{Resultados}

\section{Caracterización anatómica}

\section{Características macroscópicas}

Las características macroscópicas se presentan en la tabla 2 y en la figura 3. Por su veteado pronunciado y color es una madera con potencial para usos decorativos.

TABLA 2. Características macroscópicas de la madera de Schinus molle L.

\begin{tabular}{ll}
\hline \multicolumn{1}{c}{ Característica } & \multicolumn{1}{c}{ Descripción } \\
\hline Color & Albura rosada (5YR 7/3), Duramen café (10YR 5/3). \\
Olor & No característico \\
Sabor & No característico \\
Brillo & Medio \\
Hilo & Inclinado a entrecruzado \\
Textura & Media \\
Veteado & Pronunciado \\
Visibilidad de elementos constitutivos & Zonas de crecimiento a simple vista, los radios y poros visibles con lupa. \\
\hline
\end{tabular}




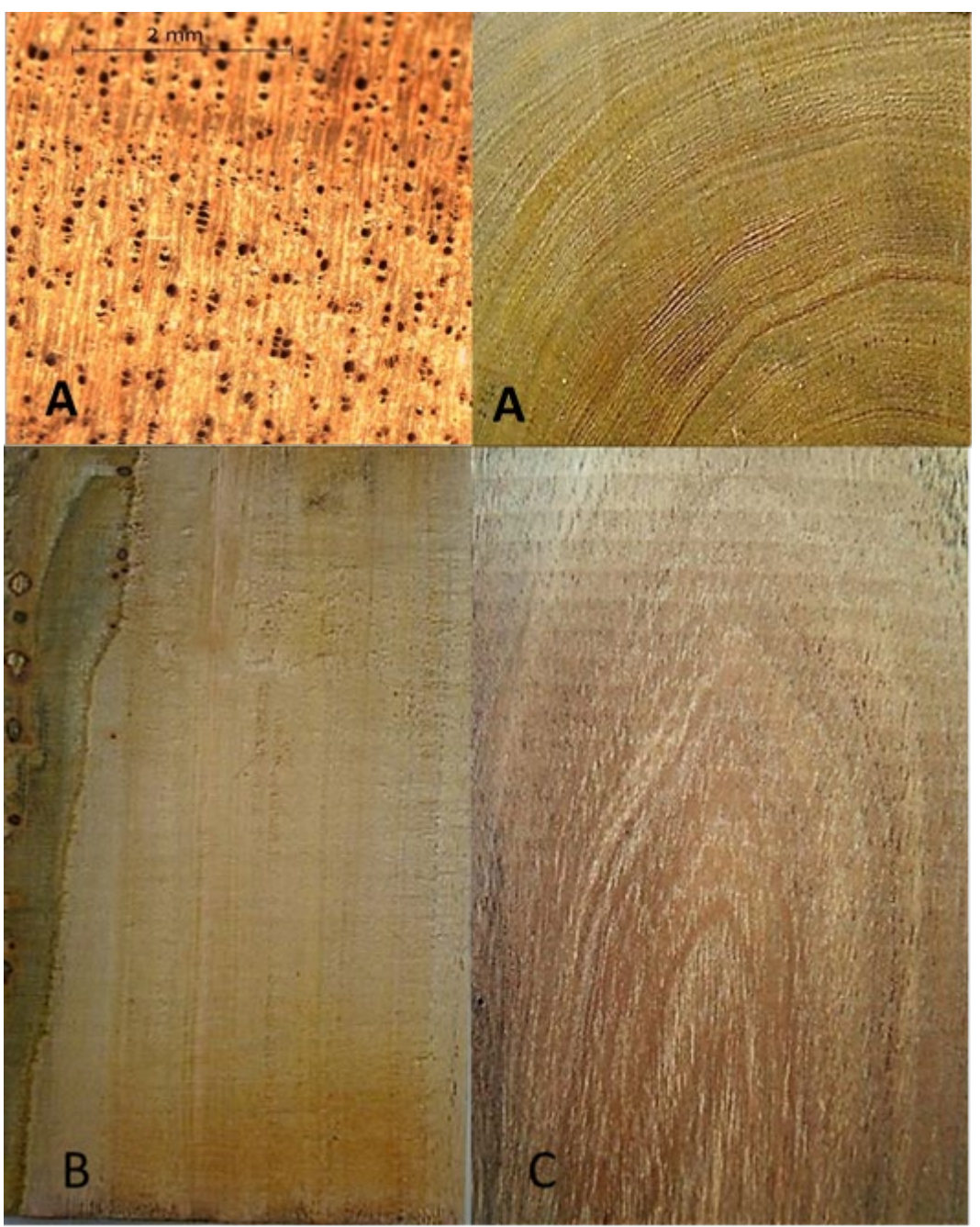

FIGURA 3. Cortes típicos macroscópicos de la madera de Schinus molle. A: corte transversal, B: Corte radial, C: corte tangencial

\section{Características microscópicas}

Porosidad semidifusa, los poros generalmente múltiples, en líneas radiales en su mayoría de 2 a 3 elementos de vaso con inclusiones (gomas), los radios son uniseriados, biseriados, los más abundantes, triseriados y heterogéneos con cristales, fibras septadas (Fig. 4), parénquima axial apotraqueal difuso y paratraqueal escaso (Fig. 5). Vasos con puntuaciones alternas, algunos presentaron ápice, placa de perforación simple. La medición de la longitud, diámetro total y del lumen de fibras se muestra en la figura 6. La clasificación de cada una de las características evaluadas se presenta en la tabla 3.

\section{Proporción de elementos constitutivos}

La proporción de elementos constitutivos de $S$. molle se clasificó de acuerdo con Kollmann (1959), las fibras presentaron la mayor proporción en la madera, la cantidad de parénquima axial fue muy escasa en relación con la cantidad de vasos, fibras y radios (Tabla 4.)

\section{Índices de calidad de pulpa}

En la tabla 5 se presentan los valores que fueron obtenidos para cada uno de los índices de calidad de pulpa y su clasificación. La Relación de Runkel indica la calidad de las fibras para la elaboración de papel. 

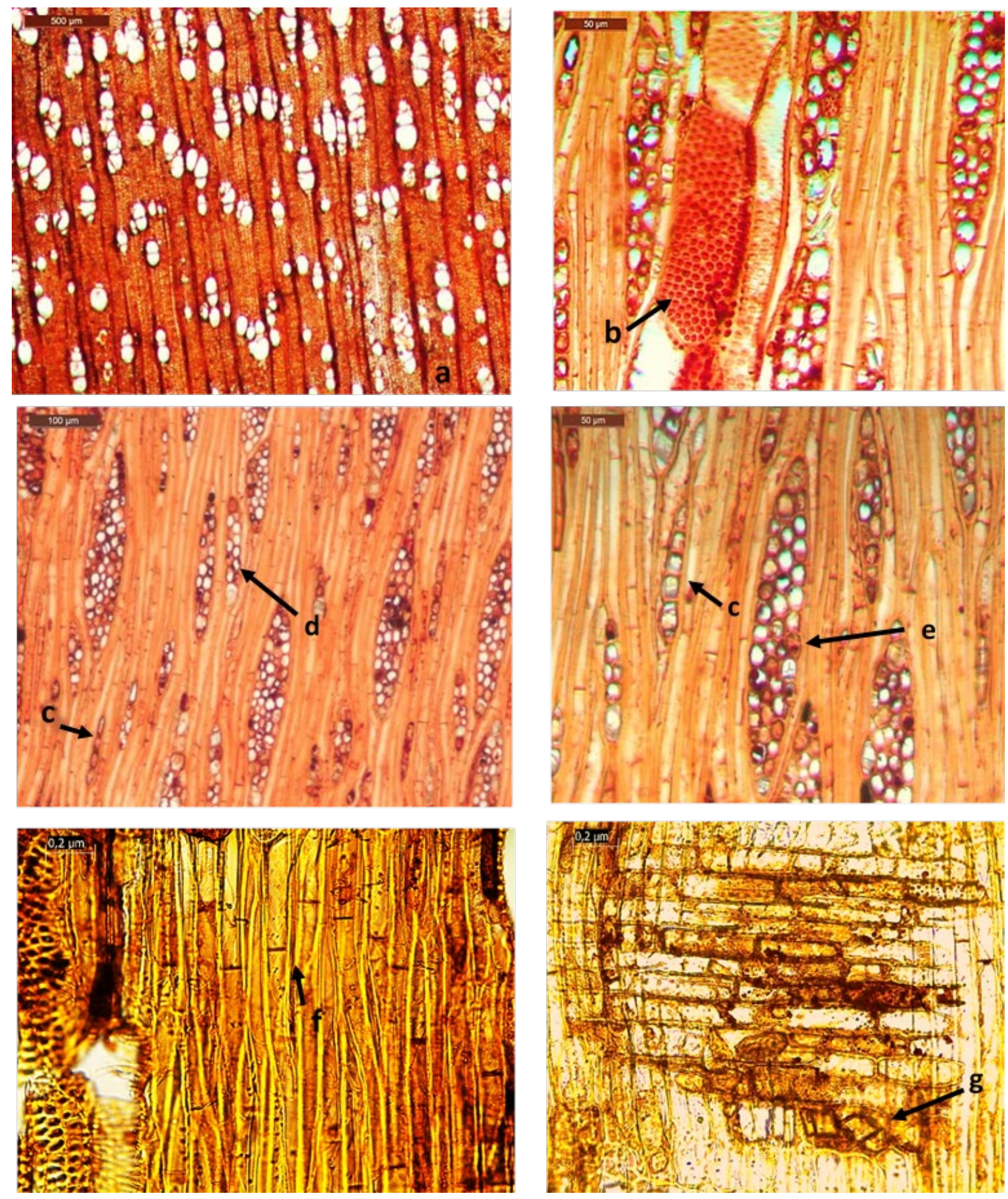

FIgURA 4. Características anatómicas microscópicas de la madera de Shinus molle.

a: porosidad semidifusa , b: puntuaciones alternas en elemento de vaso, c: radios uniseriados, d: radios biseriados e: radios triseriados , f: fibras septadas, g: cristales 

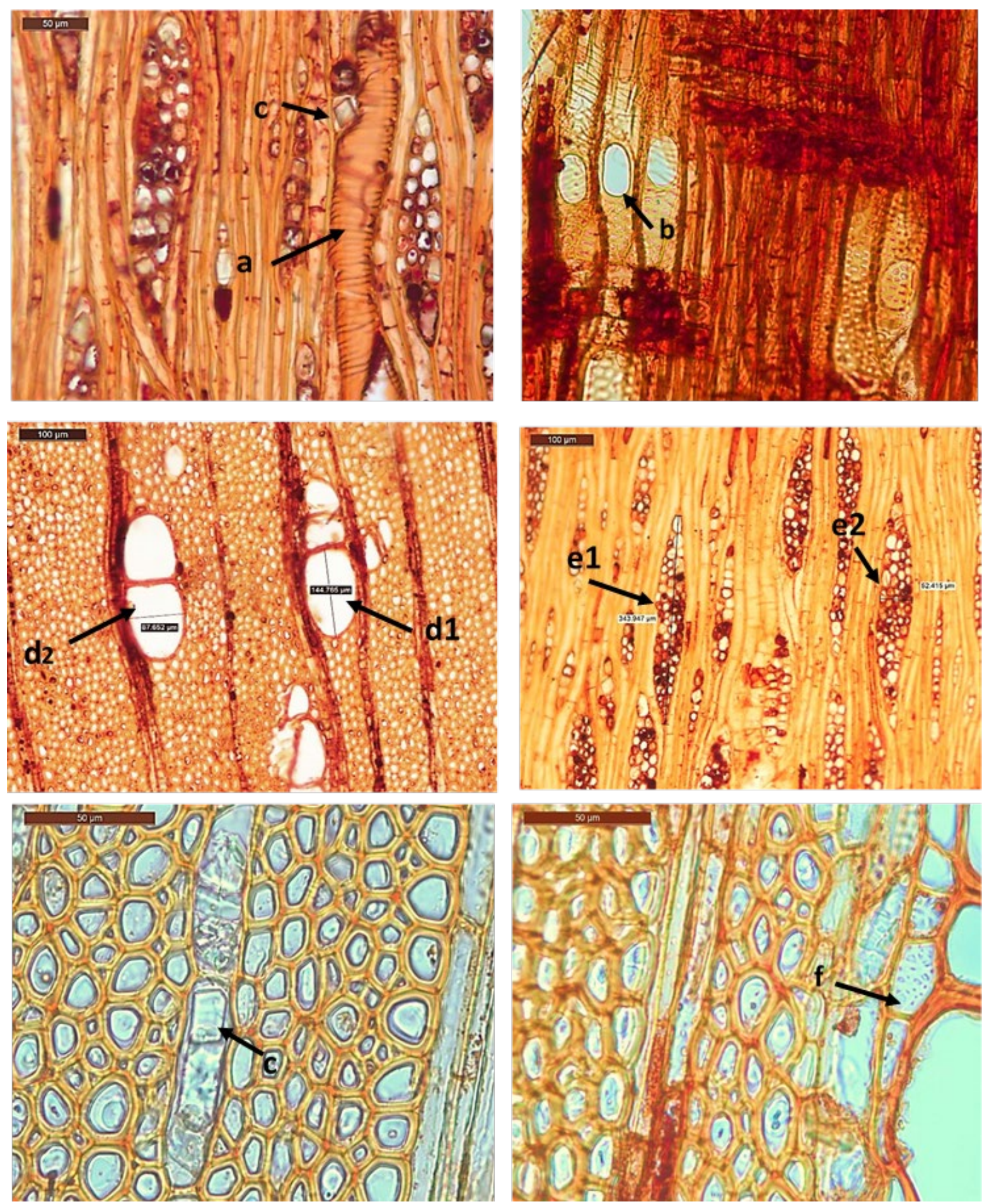

FIGURA 5. Características anatómicas de la madera de Schinus molle (continuación).

Elementos de vaso: a. engrosamiento espiralado, b. placa de perforación simple, dl. diámetro radial, d2. diámetro tangencial. Radios medulares: el. longitud, e2. ancho, f. parénquima paratraqueal escaso y c. cristales. 

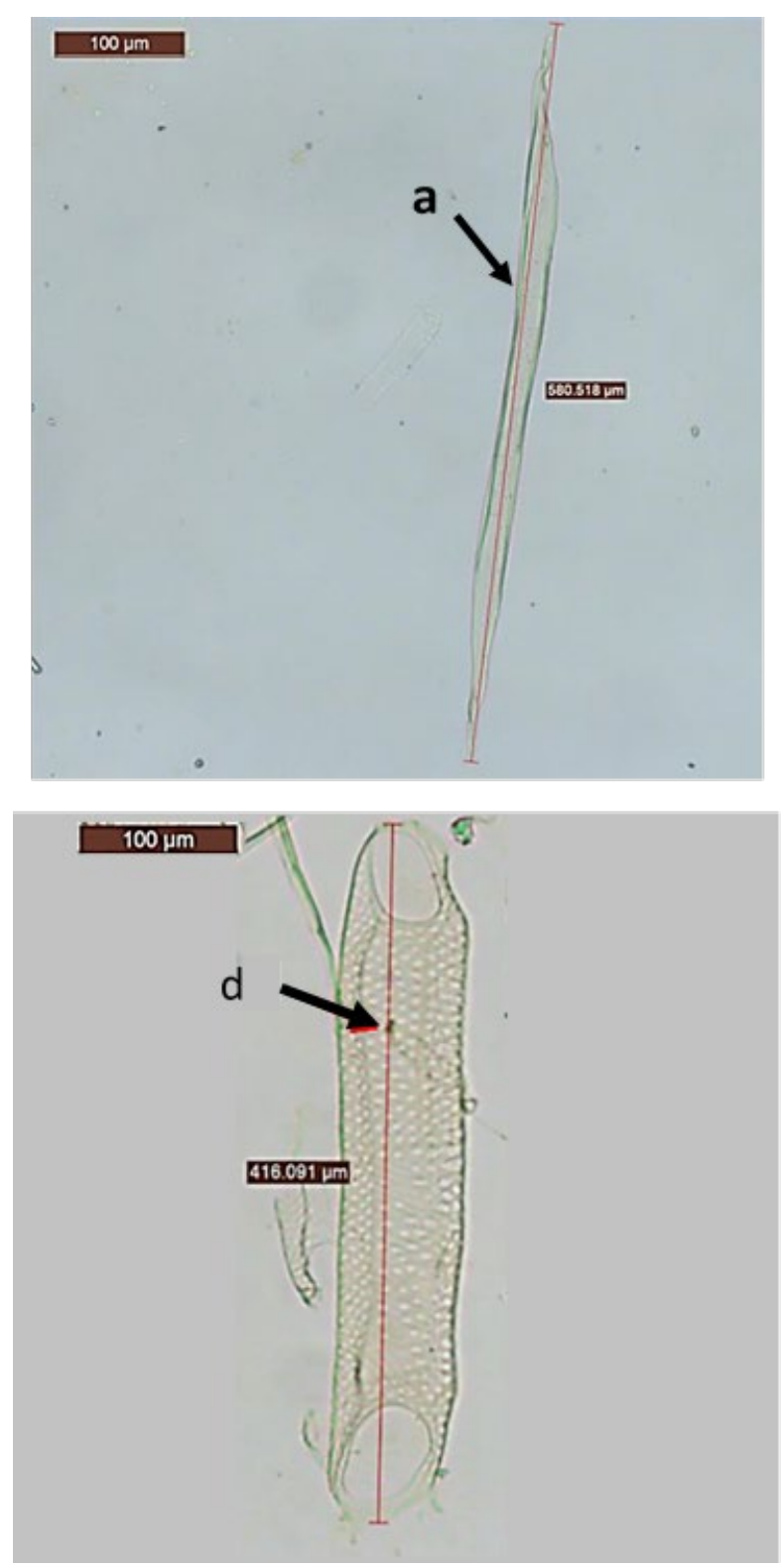

Figura 6. Material macerado de la madera de Schinus molle.

Fibras a: Iongitud, b: diámetro total, c: diámetro de lumen y d: Iongitud de elemento de vaso
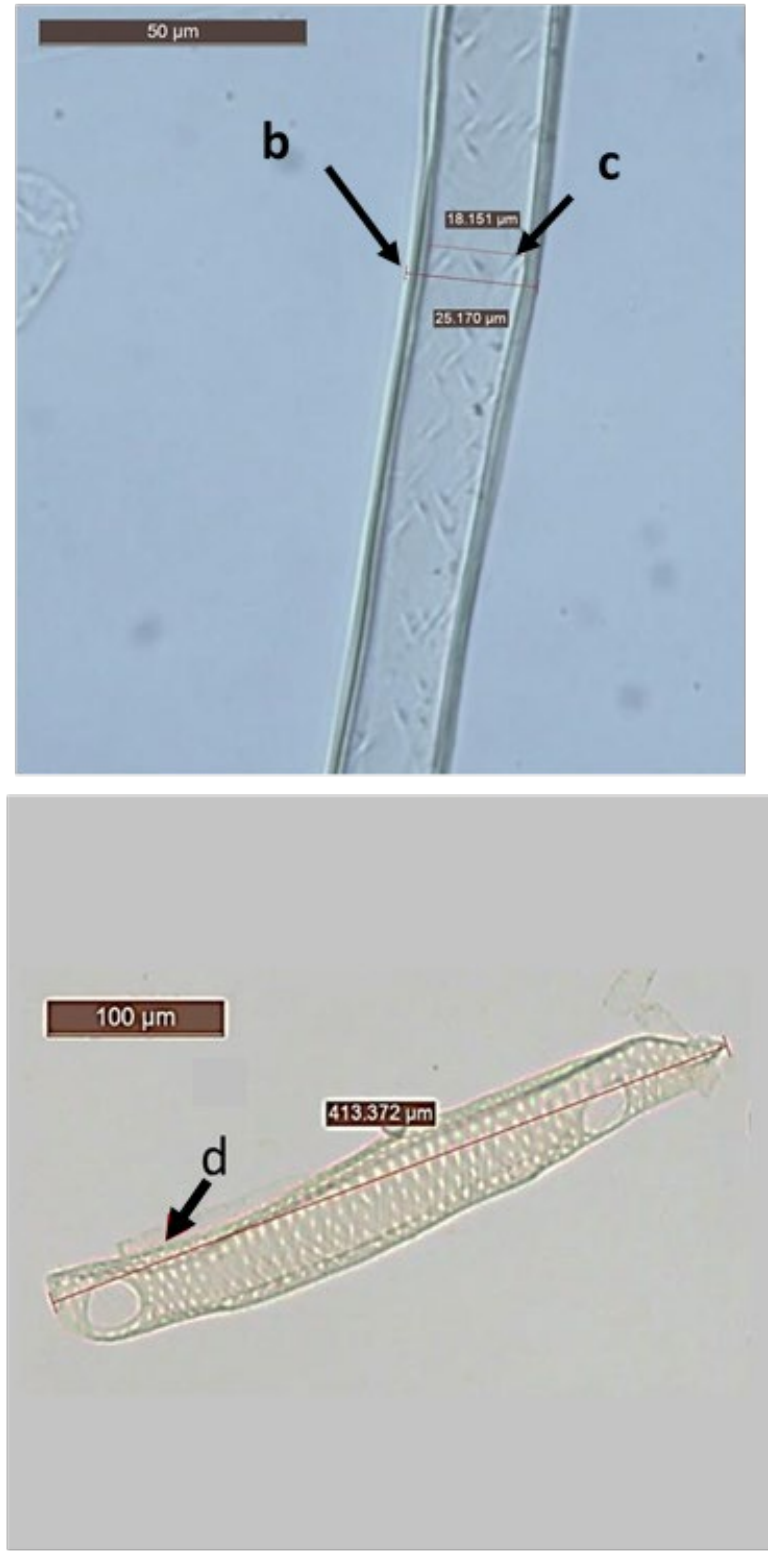

\section{Propiedades físicas}

\section{Contenido de humedad}

El contenido de humedad en condición verde se determinó en la albura y en el duramen de los cuatro árboles, en albura el promedio fue de $106 \%$ y en duramen de $83 \%$. Considerando el promedio de albura y duramen el contenido verde de humedad resulto de 95\%. También se calcularon el contenido de humedad saturado y el contenido de humedad en equilibrio, los cuales fueron de $119.9 \%$ y de $16.4 \%$ respectivamente

\section{Densidad de la madera}

La densidad básica promedio de la especie fue de 0.54, se clasificó como semipesada (Vignote y Jiménez, 1996) (Tabla 6). 
TABLA 3. Características microscópicas de la madera de Schinus molle L.

\begin{tabular}{|c|c|c|c|c|c|c|c|}
\hline Variable & $\mathrm{n}$ & Mínima & Media & Máxima & D.E. & $\begin{array}{l}\text { Coeficiente de } \\
\text { variación }\end{array}$ & $\begin{array}{l}\text { Clasificación } \\
\text { (Tortorelli, 1956) }\end{array}$ \\
\hline Fibras & 320 & & & & & & \\
\hline Longitud ( $\mu \mathrm{m})$ & & 475.351 & 755.002 & 1110.184 & 128.174 & 0.170 & Medianas \\
\hline Diámetro total ( $\mu \mathrm{m})$ & & 12.567 & 23.990 & 40.297 & 4.872 & 0.203 & Finas \\
\hline $\begin{array}{l}\text { Diámetro del lumen } \\
(\mu \mathrm{m})\end{array}$ & & 7.780 & 16.395 & 30.283 & 4.074 & 0.248 & --- \\
\hline $\begin{array}{l}\text { Grosor de la pared } \\
\text { celular }(\mu \mathrm{m})\end{array}$ & & 1.240 & 3.798 & 7.430 & 1.084 & 0.285 & Delgada \\
\hline Vasos & 480 & & & & & & \\
\hline Longitud ( $\mu \mathrm{m})$ & & 121.806 & 302.094 & 560.975 & 65.514 & 0.217 & Cortos \\
\hline $\begin{array}{l}\text { Diámetro radial } \\
(\mu \mathrm{m})\end{array}$ & & 17.455 & 44.118 & 81.978 & 16.281 & 0.369 & --- \\
\hline $\begin{array}{l}\text { Diámetro tangencial } \\
(\mu \mathrm{m})\end{array}$ & & 18.046 & 40.815 & 71.585 & 11.607 & 0.284 & --- \\
\hline Poros por mm² & & 24 & 40.3 & 72 & 14.59 & 0.361 & $\begin{array}{l}\text { Extremadamente } \\
\text { numerosos }\end{array}$ \\
\hline Radios & 1713 & & & & & & \\
\hline Altura ( $\mu \mathrm{m})$ & & 22.41 & 265.690 & 547.705 & 93.962 & 0.354 & Bajos \\
\hline Ancho ( $\mu \mathrm{m})$ & & 10.537 & 35.151 & 64.516 & 11.485 & 0.327 & Moderadamente angostos \\
\hline Radios por mm² & & 17 & 22.5 & 25 & 2.94 & 0.131 & $\begin{array}{l}\text { Extremadamente } \\
\text { numerosos }\end{array}$ \\
\hline
\end{tabular}

n= número de mediciones

D.E.= desviación estándar

TABLA 4. Proporción de elementos constitutivos de la madera de Schinus molle L.

\begin{tabular}{|c|c|c|c|c|c|}
\hline \multirow{2}{*}{ Tipo de elemento } & \multicolumn{4}{|c|}{ Valor (porcentaje) } & \multirow{2}{*}{ Clasificación } \\
\hline & Mínimo & Media & Máximo & D.E. & \\
\hline Vaso & 6.12 & 9.89 & 14.67 & 2.095 & Media \\
\hline Fibra & 74.94 & 81.40 & 87.26 & 2.688 & Mayor \\
\hline Rayo & 5.31 & 8.71 & 17.62 & 2.944 & Media \\
\hline Parénquima axial & 0.80 & 1.34 & 1.81 & 2.533 & Menor \\
\hline
\end{tabular}


TABLA 5. Índices de calidad de pulpa de la madera de Schinus molle L.

\begin{tabular}{lccccc}
\hline \multirow{2}{*}{ Índice de calidad de pulpa } & Mín. & Med. & Máx. & D.E. & Clasificación \\
\hline Coeficiente de rigidez & 0.137 & 0.319 & 0.579 & 0.078 & - \\
Coeficiente de flexibilidad & 0.421 & 0.681 & 0.863 & 0.078 & - \\
Índice de esbeltez & 15.997 & 32.478 & 62.796 & 7.462 & - \\
Relación de Runkel & 0.080 & 0.489 & 0.689 & 0.094 & Muy bueno (Tamarit, 1996) \\
\hline
\end{tabular}

D.E. = Desviación estándar

TABLA 6. Densidad de la madera de Schinus molle L.

\begin{tabular}{cccccc}
\hline Valor & $\begin{array}{c}\text { Densidad } \\
\text { verde }\end{array}$ & $\begin{array}{c}\text { Densidad a h\% de } \\
\text { contenido de } \\
\text { humedad }\end{array}$ & Densidad normal & Densidad básica & Densidad anhidra \\
\hline Mínima & 0.984 & 0.52 & 0.51 & 0.43 & 0.49 \\
Media & 1.41 & 0.69 & 0.69 & 0.54 & 0.64 \\
Máxima & 1.93 & 0.98 & 0.97 & 0.73 & 0.86 \\
D.E. & 0.0290 & 0.0861 & 0.0831 & 0.0521 & 0.0655 \\
Clasificación & & & & Semipesada & (Vignote y \\
\end{tabular}

D.E. = Desviación estándar

\section{Contracción}

El porcentaje de contracción de la madera es importante en el proceso de secado, porque de ello dependerá el tiempo y temperatura de dicho proceso para evitar deformaciones, grietas o rajaduras. Los valores obtenidos de contracción radial, tangencial y volumétrica de $S$. molle se presentan en la tabla 7.

En la tabla 8 se presentan los valores de punto de saturación de la fibra, coeficiente de contracción volumétrica y relación de anisotropía de $S$. molle.

\section{Poder calorífico}

En México se ha considerado una madera poco efectiva como leña, por los valores obtenidos en el presente estudio, esta especie puede ser una opción viable para utilizarla como combustible. En la tabla 9 se observan los valores de poder calorífico obtenidos.

\section{Propiedades mecánicas}

En la tabla 10 se muestran las propiedades mecánicas que fueron obtenidas a parir de valores de densidad básica.

\section{DISCUSIÓN}

\section{Anatomía}

Las características anatómicas macroscópicas de la madera de pirú son similares a lo encontrado por Islas (1986) a excepción del veteado, que para este estudio es pronunciado, también coincide en el color con Luna (2012) y Schulte (1992). Este último autor menciona que existen 3 anillos o zona de crecimiento en $2.5 \mathrm{~cm}$, en tanto Crivellaro y Schweingruber (2013) mencionan que los anillos son distinguibles por líneas de fibras. Luna (2012) menciona algunas características macroscópicas de S. longifolia (Lindl.) Speg. var. longifolia que presenta albura blanquecina y duramen castaño ocráceo encarnado; anillos demarcados; textura fina y grano oblicuo a entrelazado. 
TABLA 7. Contracciones de la madera de Schinus molle L.

\begin{tabular}{|c|c|c|c|}
\hline Contracción & Parcial (\%) & Total (\%) & $\begin{array}{c}\text { Clasificación } \\
\text { Fuentes-Talavera et al. } \\
\text { (2002) }\end{array}$ \\
\hline \multirow{3}{*}{ Radial } & Min. $=0.611$ & Min. $=1.285$ & \multirow{3}{*}{$\begin{array}{l}\text { Parcial }=\text { Pequeña } \\
\text { Total }=\text { Moderada }\end{array}$} \\
\hline & Med. $=2.070$ & Med. $=4.186$ & \\
\hline & Máx. = 9.545 & Max. $=12.301$ & \\
\hline \multirow{3}{*}{ Tangencial } & Min. $=1.830$ & Min. $=4.984$ & \multirow{3}{*}{$\begin{array}{l}\text { Parcial = Ligeramente } \\
\text { grande } \\
\text { Total = Ligeramente grande }\end{array}$} \\
\hline & Med. $=5.504$ & Med. $=9.029$ & \\
\hline & Max. $=14.496$ & Max. $=17.52$ & \\
\hline \multirow{3}{*}{ Axial } & Min. = 0.001 & Min. $=0.063$ & \multirow{3}{*}{------- } \\
\hline & Med.= 0.310 & Med. $=0.612$ & \\
\hline & Max.1.097 & Max.= 1.861 & \\
\hline \multirow{3}{*}{ Volumétrica } & Min. $=5.016$ & Min. $=11.744$ & \multirow{3}{*}{$\begin{array}{c}\text { Total = Media (Vignote y } \\
\text { Jiménez, 1996) }\end{array}$} \\
\hline & Med. $=9.238$ & Med. $=14.983$ & \\
\hline & Max. $=20.922$ & Max. = 26.681 & \\
\hline
\end{tabular}

Min: mínimo, Med: media, Max: máximo

TABLA 8. Punto de saturación de la fibra, coeficiente de contracción volumétrica y relación de anisotropía de la madera de Schinus molle L.

\begin{tabular}{|c|c|c|c|c|c|}
\hline \multirow{2}{*}{ Propiedad } & \multicolumn{4}{|c|}{ Valor } & \multirow{2}{*}{ Clasificación } \\
\hline & Mínimo & Media & Máximo & D.E. & \\
\hline PSF & 21.78 & 30.55 & 54.04 & 4.977 & $\begin{array}{l}\text { Normal (Novelo, } \\
\text { 1964). }\end{array}$ \\
\hline $\mathrm{CCV}$ & 0.38 & 0.49 & 0.65 & 0.046 & $\begin{array}{l}\text { Medio } \\
\text { (Fuentes,1998) }\end{array}$ \\
\hline R.A. & 0.66 & 2.20 & 6.78 & 0.785 & $\begin{array}{l}\text { Media } \\
\text { (Fuentes,1998) }\end{array}$ \\
\hline
\end{tabular}

D.E.= desviación estándar, PSF = Punto de saturación de la fibra, CCV = Coeficiente de contracción volumétrica, R.A. = Relación de anisotropía.

TABLA 9. Valores de poder calorífico de la madera de Schinus molle L

\begin{tabular}{cc}
\hline Valor & Poder calorífico $\left(\mathrm{MJ} \mathrm{Rg} \mathrm{kg}^{-1}\right.$ \\
\hline Mínimo & 16.787 \\
Media & 17.246 \\
Máximo & 17.626 \\
Desviación estándar & 60.617 \\
\hline
\end{tabular}


TABLA 10. Propiedades mecánicas calculadas a partir de la densidad básica y la densidad normal de la madera de Schinus molle L.

\begin{tabular}{|c|c|c|c|c|}
\hline Ensayo & Madera verde & $\begin{array}{c}\text { Clasificación } \\
\text { (Echenique y } \\
\text { Plumptre, } \\
\text { 1994) }\end{array}$ & $\begin{array}{c}\text { Madera a } 12 \% \text { de } \\
\mathrm{CH}\end{array}$ & $\begin{array}{c}\text { Clasificación } \\
\text { (Dávalos y } \\
\text { Bárcenas, } \\
\text { 1999) }\end{array}$ \\
\hline \multicolumn{5}{|l|}{ Flexión estática } \\
\hline Esfuerzo al límite de proporcionalidad (MPa) & 32.554 & --- & 72.410 & --- \\
\hline Módulo de ruptura & 56.172 & Baja & 111.433 & Alto \\
\hline Módulo de elasticidad (MPa) & 8786 & Bajo & 13321 & Alto \\
\hline Impacto & 9.659 & ----- & 12.309 & ---- \\
\hline \multicolumn{5}{|l|}{ Compresión paralela a la fibra } \\
\hline Esfuerzo al límite de proporcionalidad (MPa) & 19.547 & Muy baja & 41.627 & ---- \\
\hline Esfuerzo máximo (MPa) & 25.057 & ----- & 58.040 & Alto \\
\hline Módulo de elasticidad (MPa) & 10834 & ----- & 16080 & ----- \\
\hline \multicolumn{5}{|l|}{ Compresión perpendicular a la fibra } \\
\hline Esfuerzo al límite de proporcionalidad (MPa) & 5.170 & Baja & 13.852 & Muy alto \\
\hline \multicolumn{5}{|l|}{ Dureza } \\
\hline Lateral (kN) & 4.158 & Media & 9.264 & Media \\
\hline Extrema (kN) & 3.802 & Baja & 7.276 & Alta \\
\hline
\end{tabular}

Respecto al tipo de porosidad, la encontrada en este estudio coincide con Islas (1986), pero no con Crivellaro Schweingruber (2013), Schulte (1992) y Moglia y Gimenez (1998) quienes observaron porosidad difusa.

El número de vasos observado en este estudio (24 $\mathrm{mm}^{-2}$ a $\left.72 \mathrm{~mm}^{-2}\right)$ es diferente al que encontró Schulte (1992) $\left(28.8 \mathrm{~mm}^{-2}\right)$ y al que encontraron Islas (1986) (80 $\mathrm{mm}^{-2}$ a $\left.120 \mathrm{~mm}^{-2}\right)$, Crivellaro y Schweingruber (2013) (200 $\mathrm{mm}^{-2}$ ) y Moglia y Gimenez (1998) (51.7 $\left.\mathrm{mm}^{-2}\right)$ Así mismo, el diámetro tangencial es menor en este estudio $(41 \mu \mathrm{m})$ en comparación con el hallado por Islas (1986): $27 \mu \mathrm{m}$ a 220 $\mu \mathrm{m}$, Schulte (1992): $68.8 \mu \mathrm{m}$, y Moglia y Gimenez (1998): $120 \mu \mathrm{m}$. El parénquima axial coincide con el observado por Crivellaro y Schweingruber (2013), Islas (1986) y Schulte (1992). En relación con las fibras, Crivellaro y Schweingruber (2013) e Islas (1986) observaron fibras libriformes y Schulte (1992), fibras libriformes y esporádicamente septadas, como en este estudio.

Los radios, los cristales romboidales, las sustancias orgánicas de color café rojizo y los elementos de vaso con pared espiralada son similares a lo observado por Schulte (1992), Islas (1986), Crivellaro y Schweingruber (2013) y Moglia y Gimenez (1998).

\section{Proporción de elementos constitutivos}

Las fibras son el elemento de mayor proporción en la madera, no se encontraron estudios que estimaran porcentaje de elementos constitutivos para la madera de $S$. molle La cantidad de parénquima axial fue muy pequeña en relación con la cantidad de vasos, fibras y radios. León (2010) registró, para Protium crenatum, una densidad básica de $0.55,20.35 \%$ de vasos, $62.15 \%$ de fibras, $13.22 \%$ de 
parénquima y $4.28 \%$ de radios; se puede comparar con $S$. molle que presenta una densidad básica de 0.54.

Los índices de calidad de pulpa para papel

Los índices calculados para $S$. molle en el presente estudio son similares a los hallados por Tamarit (1996). El coeficiente de flexibilidad deducido indica que las fibras de $S$. molle se caracterizan por ser fibras parcialmente colapsadas, con buena superficie de contacto y buena unión fibra con fibra. Así mismo, de acuerdo con la relación de Runkel, esta madera es considerada como muy buena para elaborar papel.

\section{Propiedades físicas}

La densidad básica medida en este estudio (0.54) es similar a la obtenida en estudios anteriores, a pesar de la diferencia en condiciones ambientales y espaciales (longitud, latitud y altitud) de donde fue colectado el material. Por ejemplo, Schulte (1992) encontró 0.55, Crivellaro y Schweingruber (2013) 0.50. Sin embargo, Luna (2012) observó un valor muy diferente: 0.995. Este mismo autor encontró, para Schinus terebinthifolia, una densidad de 0.55; para Schinus fasciculatus (Griseb.) I.M. Johnst., 1.005 y, para S. longifolia (Lindl.) Speg. var. longifolia, $0.80-0.88$.

Los porcentajes de contracción tangencial, radial y volumétrica son inferiores a los observados en $S$. molle por Schulte (1992), el valor de anisotropía de este estudio es superior al 1.88 calculado por este mismo autor en ese mismo estudio. La relación de anisotropía observada para la madera de $S$. molle la clasifica como de mala calidad. Se puso especial atención en el punto de saturación de la fibra determinado en el presente estudio; este se consideró para tomar las precauciones necesarias en el secado (como secuelas de secado lentas), con la finalidad de evitar deformaciones y separación de fibras durante dicho proceso.

\section{Poder calorífico}

El poder calorífico de las maderas anhidras varía poco por lo que se puede dar un valor medio de $18.84 \mathrm{MJ} \mathrm{kg}^{-1}$, en términos generales, para la técnica de combustión
Kollmann (1959). Espinoza, Vera, Carrillo y Rodríguez (1989) indican que el poder calórico promedio de Acacia retinoides es de $19.60 \mathrm{MJ} \mathrm{kg}^{-1}$, el cual es un valor alto ya que, según la Organización de las Naciones Unidas para la Agricultura y la Alimentación [FAO] (1983), arriba de 18.84 MJ kg-1 (4500 kcal kg-1), se consideran altos. Juárez y Novara (2007) calcularon un poder calórico de 16.32 MJ $\mathrm{kg}^{-1}$ para la especie de Schinus areira.

Schulte (1992) señala que $S$. molle tiene un buen poder calorífico y que la combustión lenta con la uniforme emisión de calor la vuelven especialmente apropiada para procesos de producción de pan, cerveza y chicha (bebida derivada de la fermentación de maíz); mayormente se utilizó como combustible (leña) para fundir metales que en ese entonces se explotaban. El S. molle sigue siendo fuente de leña, en menor proporción es usada para la fabricación de herramientas y hasta muebles (Schulte, 1992).

A pesar de que en México se le ha considerado una madera poco efectiva como leña, por los valores obtenidos en el presente estudio, esta especie puede ser una opción viable para ser usada como combustible.

\section{Propiedades mecánicas}

Respecto a las propiedades mecánicas, los valores de dureza lateral y extrema en condición verde hallados en este estudio son inferiores a los obtenidos por Schulte (1992), aunque dicho autor no especifica en qué condición se realizaron las pruebas mecánicas. De manera general, los valores obtenidos para cada una de las categorías tanto en condición verde como a $12 \%$ de contenido de humedad, clasifican a la madera como media a alta en cuanto a resistencia. Así mismo Luna (2012) clasifica a S. molle como madera semidura, Schinus fasciculatus (Griseb.) I.M. Johnst., madera muy dura y S. longifolia (Lindl.) Speg. var. longifolia como madera semidura, resistente a la flexión.

Así mismo, Schulte (1992) indica que esta especie es conocida desde el periodo prehispánico; los incas, teniendo un conocimiento empírico sobre la dureza de su madera, la usaban para fabricar herramientas y utensilios. 


\section{CONCLUSIONES}

Por sus características anatómicas macroscópicas, como color y veteado, es una especie viable para elaboración de muebles o mangos para herramienta de jardinería. De acuerdo con la relación de Runkel, la calidad de pulpa para papel de la madera de pirú es buena, por lo que no se descarta como una posible especie para obtener pulpa para papel. Considerando la densidad básica, se puede utilizar para la fabricación de pisos.

En México se le ha considerado una madera poco efectiva como leña, pero por los valores del poder calorífico, esta especie puede ser una opción viable para ser usada como combustible.

\section{REFERENCIAS}

Alba, G. A., Bonilla, R. P., \& Arroyo, A. J. (2009). Actividad cicatrizante de una pomada con aceite esencial de Schinus molle L. "molle" en ganado vacuno con heridas infectadas y en ratones. Ciencia e investigación, 12(1), 29-36. doi: 10.15381/ci.v12i1.3384

Anderson, P. (s.f.). Parte II: Species Descriptions-Scbinus molle L. Bolivia, Peace corps. 710-712.

Brandt, R., Zimmermann, H., Hensen, I., Castro, J. C. M., \& Rist, S. (2012). Agroforestry species of the Bolivian Andes: an integrated assessment of ecological, economic and socio-cultural plant values. Agroforestry systems, 86(1), 1-16. doi: 10.1007/s10457-0129503-y

Brown, H. P., \&Panshin, A. J. (1980). Textbook of Wood technology: The physical, mechanical and chemical properties of the commercial woods of the United States. Nueva York, Estados Unidos de America: McgrawHill Book Company.

Calderón de R, G. \& Rzedowski, J. (2001). Flora fanerogámica del V alle de México. Michoacán, México. Instituto de Ecología. A.C.-Comisión Nacional para el Conocimiento y Uso de la Biodiversidad.

Camacho, M. F. (1985). Identificación del mecanismo que inhibe la germinación en Schinus molle L. y forma de eliminarlo. Revista Ciencia Forestal, 10(55), 35-49.

Camacho, M. F., \& Ramírez, P. M. (1987). Dormición química de semillas de pirú (Schinus molle L.) en tres tipos de siembra. Revista Ciencia Forestal, 12(62), 15-31.

Carrere, R. (2009). Anacahuita (Schinus molle): la indígena más popular. Colección del Grupo Guayabira Sobre Especies Indígenas, (15). Recuperado de http://www.guayubira.org.uy/monte/Anacahuita.pdf
Cardoso, M. B., Ladio, A. H., \& Lozada, M. (2012). The use of firewood in a Mapuche community in a semi-arid region of Patagonia, Argentina. Biomass and Bioenergy, 46, 155-164. doi: 10.1016/j.biombioe.2012.09.008

Cardoso, M. B., Ladio, A. H., \& Lozada, M. (2013). Fuelwood consumption patterns and resilience in two rural communities of the Northwest Patagonian steppe, Argentina. Journal of Arid Environments, 98, 146-152. doi: 10.1016/j.jaridenv.2012.09.013

Cardoso, M. B., Ladio, A. H., Dutrus, S. M., \& Lozada, M. (2015). Preference and calorific value of fuelwood species in rural populations in northwestern Patagonia. Biomass and Bioenergy, 81, 514-520. doi: 10.1016/j.biombioe.2015.08.003

Corkidi, L., Cacho, S., \& Búrquez, A. (1991). Dispersión del pirú (Scbinus molle L., Anacardiaceae) por aves en Teotihuacán, México. Acta Botánica Mexicana, 15, 17-22. doi: 10.21829/abm15.1991.617

Crivellaro, A., \& Schweingruber, F. H. (2013). Atlas of wood, bark and pith anatomy of Eastern Mediterranean trees and shrubs: with a special focus on cyprus. Springer Science \& Business Media.

Dávalos S., R., \& Bárcenas P., G. (1999). Clasificación de las propiedades mecánicas de las maderas mexicanas en condición "seca". Madera y Bosques, 5(1) 61-69. doi: 10.21829/myb.1999.511355

dos Santos C., A., de Souza A., M., da Silva P., L., dos Santos P., D., Nalesso S., M., de Almeida C., D., \& Alves de S., M. (2015). Volatiles composition and extraction kinetics from Schinus terebinthifolius and Schinus molle leaves and fruit. Revista Brasileira de Farmacognosia, 25(4), 356-362. doi: 10.1016/j.bjp.2015.07.003

Echenique, R., \& Plumptre, A. (1994). Guia para el uso de maderas de México y Belice. Guadalajara, México: Universidad de Guadalajara.

Espinoza A., J., Vera C., G., Carrillo A., F., \& Rodríguez F., C. (1989). Evaluación de plantaciones de Acacia retinoides Scblecbt. con fines de producción de leña combustible. Memorias de la Primera Reunión Nacional Sobre Dendroenergía. 8 y 9 de noviembre de 1989. Universidad Autónoma Chapingo, México. pp. 27-39.

Fuentes, M. (1998). Propiedades tecnológicas de las maderas mexicanas de importancia en la construcción. Revista Chapingo, serie ciencias Forestales y del ambiente, 4(1), 221-229.

Fuentes-Talavera, F., Silva-Guzmán, J., Lomelí-Ramírez, M., Richter, H., \& Sanjuan-Dueñas, R. (2002) Comportamiento higroscópico de la madera de Persea americana var. guatemalensis Mill (Hass). Revista Chapingo, serie ciencias Forestales y del Ambiente, 8(1), 49-56.

Goldstein, D., \& Coleman, R. (2004). Schinus molle L. (Anacardiaceae) Chicha production in the Central Andes. Economic Botany, 58(4), 523529. doi: 10.1663/0013-0001(2004)058[0523:SMLACP]2.0.CO;2 
Instituto Nacional para el Federalismo y el Desarrollo Municipal [INFDM] (2002). Enciclopedia de los Municipios de México, Gobierno del Estado de Hidalgo. Recuperado de: http://www.inafed.gob.mx/work/enciclopedia/EMM13hidalgo /index.html

Islas, A. (1986). El pirú (Schinus molle) usos y perspectivas en México. Tesis de licenciatura, Universidad Autónoma Chapingo, Texcoco, México.

Juárez, F., \& Novara, L. (2007). Flora del valle de Lerma: Anacardiaceae Lindl. Aportes Botánicos de Salta Ser. Flora, 8(6), 1-35.

Kollmann, F. (1959). Tecnología de la madera y sus aplicaciones. Madrid, España. Ministerio de Agricultura. Instituto Forestal de Investigaciones, Experiencias y Servicio de la Madera.

León, W. (2010). Anatomía y densidad o peso específico de la madera. Revista Forestal Venezolana, 54(1) 67-76.

López, A., Castro, S., Andina, M., Ures, X., Munguía, B., Llabot, J., Elder, H., Dellacassa, E., Palma, S., \& Domínguez, L. (2014). Insecticidal activity of microencapsulated Schinus molle essential oil. Industrial Crops and Products, 53, 209-216. doi: 10.1016/j.indcrop.2013.12.038

Luna, C. (2012). Distribución e importancia maderera de la familia Anacardiáceas en El Gran Chaco Argentino. Ra Ximbai, 8(3), 8395. doi: 10.35197/rx.08.03.e1.2012.09.cv

Martínez, G. (2015). Cultural patterns of firewood use as a tool for conservation: A study of multiple perceptions in a semiarid region of Cordoba, Central Argentina. Journal of Arid Environments, 121, 84-99. doi: 10.1016/j.jaridenv.2015.05.004

Martins, M., Arantes, S., Candeias, F., Tinoco, M., \& Cruz-Morais, J. (2014). Antioxidant, antimicrobial and toxicological properties of Schinus molle L. essential oils. Journal of Ethnopharmacology, 151(1), 485-492. doi: 10.1016/j.jep.2013.10.063

Mejía-Díaz, L. A., \& Rutiaga-Quiñones, J. G. (2008). Chemical composition of Schinus molle L. wood and kraft pulping process. Revista Mexicana de Ingeniería Quimica, 7(2) ,145-149.

Molina-Salinas, G., Pérez-López, A., Becerril-Montes, P., SalazarAranda, R., Said-Fernández, S., \& Waksman de Torres, N. (2007). Evaluation of the flora of Northern Mexico for in vitro antimicrobial and antituberculosis activity. Journal of Ethnopharmacology, 109(3), 435-441. doi: 10.1016/j.jep.2006.08.014

Moglia, G., \& Giménez, A. M. (1998). Rasgos anatómicos característicos del hidrosistema de las principales especies arbóreas de la región chaqueña Argentina. Forest Systems, 7(1), 53-71.

Mohareb A., S., Badawy M., E., \& Abdelgaleil S., A. (2013). Antifungal activity of essential oils isolated from Egyptian plants against wood decay fungi. Journal of wood science, 59(6), 499-505. doi: $10.1007 / \mathrm{s} 10086-013-1361-3$

Munsell, A. H. (2000). Munsell soil color charts. Munsell Color.

Navarro M., J., Borja de la R., A., \& Machuca V., R. (2005). Características tecnológicas de la madera de palo morado (Peltogyne mexicana Martínez) de tierra colorada, Guerrero, México. Revista Chapingo Serie Ciencias Forestales y del Ambiente, 11(1): 73-82.

NMX-EE-117-1981. Envase y embalaje. Determinación de peso específico aparente en maderas. Secretaría de Patrimonio y Fomento Industrial. México, D.F.

NMX-EE-167-1983. Envase y embalaje. Madera Contracción lineal. Método de prueba. Secretaría de Patrimonio y Fomento Industrial. México, D.F.

Novelo, G. (1964). Aplicaciones de la madera de cuatro especies tropicales con base a sus propiedades fisicas y mecánicas. Tesis de licenciatura. Escuela Nacional de Agricultura. Texcoco, México.

Organización de las Naciones Unidas para la Agricultura y la alimentación [FAO] (1983). Métodos simples para fabricar carbón vegetal, 41

Ramírez-Albores, J., \& Badano, E. (2013). Perspectiva histórica, sociocultural y ecológica de una invasión biológica: el caso del Pirúl (Schinus molle L., Anacardiaceae) en México. Boletín de la Red Latinoamericana para el Estudio de Especies Invasoras, 3(1), 4-15.

Rzedowski, J., \& Calderón, G. (1999). Flora del Bajío y de regiones adyacentes: Anacardiaceae. Fascículo 78. Instituto de EcologíaCentro Regional del Bajío. Pátzcuaro, Michoacán, México. doi: $10.21829 / \mathrm{fb} .285 .1999 .78$

Schulte, A. (Ed). (1992). Reforestación y agroforestería de los Andes. Uso sostenido, conservación y restauración de suelos con árboles y arbustos nativos. Apuntes sobre el Molle (Schinus molle). Cochabamba, Bolivia. Universidad Cochabamba.

Serna M., Y., Borja de la R., A., Fuentes S., M., \& Corona A., A. (2011). Propiedades tecnológicas de la madera de algarrobo (Hymenaea oblongifolia Huber.), de Bagadó-Chocó, Colombia. Revista Chapingo Serie Ciencias Forestales y del Ambiente, 17(3), 411-422. doi: 10.5154/r.rchscfa.2010.07.043

Tamarit U., J., (1996). Determinación de los índices de calidad de pulpa para papel de 132 maderas latifoliadas. Madera y Bosques, 2(2), 2941. doi: $10.21829 /$ myb.1996.221384

Tamarit U., J., \& Fuentes S., M. (2003). Parámetros de humedad de 63 maderas latifoliadas mexicanas en función de su densidad básica. Revista Chapingo Serie Ciencias Forestales y del Ambiente, 9(2), 155-164.

Tortorelli, A. (1956). Maderas y bosques de Argentina. Buenos Aries, Argentina: ACME. S.A. 
Vignote, S., \& Jiménez, F. (1996). Tecnología de la madera. Madrid, España: Ministerio de Agricultura, Pesca y Alimentación.

Villaseñor R., J. L., \& Espinosa G., F. (1998). Catálogo de malezas de México. México, D.F.: Fondo de Cultura Económica.

Whaley, Q., Beresford-Jones, D., Milliken, W., Orellana, A., Smyk, A., \& Leguía, J. (2010). An ecosystem approach to restoration and sustainable management of dry forest in southern Peru. Kew Bulletin, 65(4), 613-641. doi: 10.1007/s12225-010-9235-y

Manuscrito recibido el 16 de mayo de 2017

Aceptado el 6 de agosto de 2019

Publicado el 6 de julio de 2021
Este documento se debe citar como:

Olvera-Licona, G., Machuca, R., Borja, A., Corona, A., Zaragoza, I., Arreola, J. G., \& Jiménez, J. (2021) Xilotecnia de la madera de Schinus molle L. de una plantación forestal comercial en Hidalgo, México. Madera y Bosques, 27(1), e2711567. doi: 10.21829/myb.2021.2711567

Madera y Bosques por Instituto de Ecología, A.C. se distribuye bajo una Licencia Creative Commons Atribución-NoComercial-Compartirlgual 4.0 Internacional. 